\title{
Excited-State Dynamics of Donor-Acceptor Bridged Systems Containing a Boron-Dipyrromethene Chromophore: Interplay between Charge Separation and Reorientational Motion
}

\author{
Guillaume Duvanel, Natalie Banerji, and Eric Vauthey* \\ Department of Physical Chemistry, University of Geneva, 30 Quai Ernest-Ansermet, \\ CH-1211 Geneva, Switzerland
}

Received: February 26, 2007; In Final Form: April 12, 2007

\begin{abstract}
The excited-state dynamics of a series of electron donor-acceptor bridged systems (DABS) consisting of a boron-dipyrromethene chromophore covalently linked to a dinitro-substituted triptycene has been investigated using femtosecond time-resolved spectroscopy. The chromophores differ by the number of bromine atom substituents. The fluorescence lifetime of the DABS without any bromine atom is strongly reduced when going from toluene to polar solvents, this shortening being already present in chloroform. This effect is about 10 times weaker with a single bromine atom and negligible with two bromine atoms on the chromophore. The excited-state lifetime shortening is ascribed to a charge transfer from the excited chromophore to a nitrobenzene moiety, the driving force of this process depending on the number of bromine substituents. The occurrence of this process is further confirmed by the investigation of the excited-state dynamics of the chromophore alone in pure nitrobenzene. Surprisingly, no correlation between the charge separation time constant and the dielectric properties of the solvents could be observed. However, a good correlation between the charge separation time constant and the diffusional reorientation time of the chromophore alone, measured by fluorescence anisotropy, was found. Quantum chemistry calculations suggest that quasi-free rotation about the single bond linking the chromophore to the triptycene moiety permits a sufficient coupling of the donor and the acceptor to ensure efficient charge separation. The charge separation dynamics in these molecules is thus controlled by the reorientational motion of the donor relative to the acceptor.
\end{abstract}

\section{Introduction}

The distance between donor, $\mathrm{D}$, and acceptor, $\mathrm{A}$, is known to be a crucial parameter for the rate of photoinduced electrontransfer (ET) reactions, and for this reason, its effect on the ET dynamics has been very intensively investigated. ${ }^{1-7}$ Comparatively, the influence of the relative orientation of the reactants has been overlooked. In ET theories, both D and A are considered as spheres, and thus the only explicit geometrical factor is the distance. ${ }^{8,9}$ The effect of orientation is implicitly taken into account in the matrix element for electronic coupling, $V_{\mathrm{DA}}$, which depends on the overlap of the electronic wave functions of $\mathrm{D}$ and $\mathrm{A}$ and thus on their relative geometry. Experimentally, the importance of the mutual orientation of D and A on the ET dynamics has been evidenced in several cases, particularly in covalently linked DA pairs. ${ }^{10-22}$ Consequently, a similar influence can also be expected for intermolecular ET reactions. Although invoked in several cases, ${ }^{23-27}$ the occurrence of orientational effects in such intermolecular reactions could never be firmly established. The major reason for this is that bimolecular ET is limited by the rate of translational diffusion, which is typically of the order of $10^{10} \mathrm{M}^{-1} \mathrm{~s}^{-1}$ in nonviscous solvents. Orientational effects can only play a role once the reactants are at contact or close to contact distance. As reorientational motion is faster than translation, ET limited by rotational diffusion cannot be readily observed. Translational diffusion can be suppressed when investigating ET quenching in reacting solvents. In this case, however, the situation is

*Corresponding author. E-mail: eric.vauthey@chiphy.unige.ch. complicated by the fact that several solvent molecules can act as quencher, and thus the extraction of ET rate constant from the quenching rate constant is not straightforward..$^{25,26}$

We present here our investigation on covalently linked electron donor-acceptor bridged systems (DABS) that allow the interplay between reorientational motion and ET to be investigated. As shown in Figure 1, these DABS are composed of a boron dipyrromethene (BODIPY) derivative, 4,4-difluoro1,3,5,7-tetramethyl-4-borata-3a-azonia-4a-aza-s-indacene (TMBDY) linked in the 8-position to a dinitro-triptycene. As will be shown below, the excited BODIPY chromophore acts as electron donor, whereas the nitrobenzene units act as electron acceptors. The D and A moieties are at a fixed center-to-center distance, but their relative orientation can vary upon rotation about the single bond at the 8-position. Moreover, the electron-donating ability of the excited D can be decreased upon addition of bromine substituents.

BODIPY chromophores are characterized by an intense fluorescence in the visible region ${ }^{28,29}$ and have thus become fluorescent probes used in many different areas such as, for example, DNA sequencing, ${ }^{30,31}$ ion $^{32,33}$ and nitric oxide sensing, ${ }^{34}$ production of singlet oxygen by photosensitization, ${ }^{35}$ or single molecule detection. ${ }^{36}$ BODIPY chromophores have also been used in synthetic light-harvesting antenna, ${ }^{37}$ photonic wires ${ }^{38}$ optoelectronic gates, ${ }^{39}$ and other energy donor-acceptor systems. ${ }^{40-42}$

Photoinduced ET reactions with BODIPY dyes have already been reported. Tonimura et al. have shown that the fluorescence of BODIPY dyes can be efficiently quenched by nucleotide 


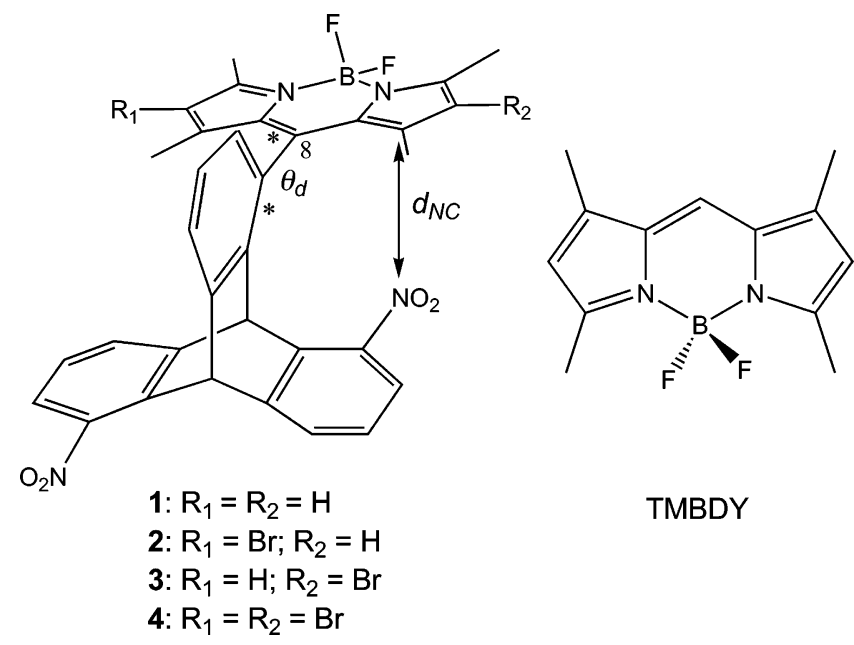

Figure 1. Chemical structure of the donor-acceptor bridged systems (DABS) and of TMBDY. The stars define the dihedral angle, $\theta_{\mathrm{d}}$.

bases. ${ }^{43}$ More recently, Hattori et al. have investigated a BODIPY substituted with a trimethoxybenzene at the 8-position. ${ }^{44}$ The resulting molecule is nonfluorescent in acetonitrile because of an ultrafast intramolecular ET from the trimethoxybenzene substituent, which acts as an electron donor, to the excited BODIPY moiety. Similarly, Qin et al. have investigated the influence of the nature of 8-substitution on the fluorescence properties of BODIPY dyes and found that substitution by electron-donating groups, such as amines, leads to a reduced fluorescence quantum yield due to the population of an intramolecular charge-transfer state from the locally excited singlet state of BODIPY..$^{45}$ Moreover, in solvents of moderate polarity a broad and structureless emission band, ascribed to charge transfer emission was observed.

The molecules investigated here differ considerably from the BODIPY derivatives discussed just above for at least two reasons: (1) the BODIPY chromophore acts here as electron donor, and (2) these are DABS, whereas the above-mentioned molecules are directly linked DA pairs. Indeed, triptycene has been shown to be equivalent to three weakly coupled $o$-xylene molecules. ${ }^{46-48}$ Thus, the benzene ring of the triptycenyl moiety directly connected to the chromophore can be considered as a bridge, whereas the nitrobenzene moieties can be regarded as two independent electron acceptors.

Additionally to these DABS, the fluorescence quenching of TMBDY alone in pure nitrobenzene as well as its reorientational dynamics in various solvents has been investigated.

\section{Experimental Section}

Steady-State Measurements. Absorption spectra were recorded on a Cary 50 spectrophotometer, while fluorescence and excitation spectra were measured on a Cary Eclipse fluorometer (5 $\mathrm{nm}$ slit) in a $1 \mathrm{~cm}$ quartz cell.

Time-Resolved Fluorescence Measurements. Excited-state lifetime measurements in the nanosecond time scale were carried out with the time-correlated single-photon counting (TCSPC) technique. Excitation was performed at a repetition rate of 40 $\mathrm{MHz}$ with $<90 \mathrm{ps}$ pulses generated by laser diodes either at $395 \mathrm{~nm}$ (Picoquant model LDH-P-C-400B) or at $469 \mathrm{~nm}$ (Picoquant model LDH-P-C-470). Fluorescence was collected at $90^{\circ}$ with a polarization at magic angle to that of the pump pulses. The detected fluorescence wavelength was selected with interference filters. Additionally, a $420 \mathrm{~nm}$ or $560 \mathrm{~nm}$ cutoff filter placed in front of the photomultiplier tube (Hamamatsu, H5783-P-01) ensured that no scattered excitation light could reach the detector, whose output was connected to the input of a TCSPC computer board module (Becker and Hickl, SPC-30012). The full width at half-maximum (fwhm) of the instrument response function (IRF) was around 200 ps. The accuracy on the lifetimes is of ca. $0.1 \mathrm{~ns}$.

Excited-state lifetime measurements in shorter time scales were performed using fluorescence up-conversion (FU). The experimental setup has been described in detail elsewhere. ${ }^{49}$ Excitation was achieved at $400 \mathrm{~nm}$ with the frequency-doubled output of a Kerr lens mode-locked Ti:Sapphire laser (Tsunami, Spectra-Physics). The output pulses centered at $800 \mathrm{~nm}$ had a duration of $100 \mathrm{fs}$ and a repetition rate of $82 \mathrm{MHz}$. The polarization of the pump beam was at magic angle relative to that of the gate pulses at $800 \mathrm{~nm}$ except for fluorescence anisotropy measurements. Experiments were carried out in a $0.4 \mathrm{~mm}$ rotating cell. The fwhm of the IRF was ca. $210 \mathrm{fs}$.

Femtosecond-resolved fluorescence anisotropy measurements were carried out with the FU setup by changing the polarization of the pump beam with respect to that of the gate beam with a half-wave plate. The anisotropy decay, $r(t)$, was reconstructed using the standard equation, $r(t)=I_{\|}(t)-I_{\perp}(t) /\left(I_{\|}(t)+2 I_{\perp}(t)\right)$, where $I_{\|}(t)$ and $I_{\perp}(t)$ are the fluorescence intensities recorded with the polarization of the pump beam set parallel and perpendicular to that of the gate beam, respectively.

Transient Absorption (TA) Measurements. Excitation at $500 \mathrm{~nm}$ was performed with a two-stage noncollinear optical parametric amplifier (NOPA, Clark-MXR), fed by the $800 \mathrm{~nm}$ output of a standard $1 \mathrm{kHz}$ amplified Ti:Sapphire system (Spectra-Physics). After recompression with a pair of prisms, the pulse duration was of the order of $50 \mathrm{fs}$. The energy per pulse at the sample was around $1 \mu \mathrm{J}$. Probing was achieved with a white light continuum obtained by focusing $800 \mathrm{~nm}$ pulses in a $\mathrm{H}_{2} \mathrm{O} / \mathrm{D}_{2} \mathrm{O}$ mixture. The probe beam was split before the sample into a pumped signal beam and an unpumped reference beam. The transmitted signal and reference beams were detected with two ORIEL Multispec 125 spectrographs equipped with a 2048 pixels CCD line (Entwicklungsbüro Stresing, Berlin). To improve sensitivity, the pump light was chopped at half the amplifier frequency, and the transmitted signal intensity was recorded shot by shot. It was corrected for intensity fluctuations using the reference beam. The transient spectra were averaged until the desired signal-to-noise ratio was achieved.

Data Analysis. Time-resolved data were analyzed by iterative reconvolution of the IRF with trial functions (sum of exponentials) using a nonlinear least-squares fitting procedure (MATLAB, The MathWorks, Inc.).

Samples. Compounds $\mathbf{1 - 4}$ and TMBDY were given by Professor A. Gossauer (Chemistry Department of the University of Fribourg) and were used as received. The solvents, acetonitrile $(\mathrm{ACN})$, chloroform, dichloromethane (DCM), 1,2-dichloroethane (DCE), and toluene (TOL) were of the highest commercially available purity and were used without further purification. Propionitrile ( $\mathrm{PrCN})$, butyronitrile $(\mathrm{BuCN})$, valeronitrile $(\mathrm{VaCN})$, benzonitrile $(\mathrm{BzCN})$, and nitrobenzene were purified as described in the litterature. ${ }^{50}$ For TCSPC measurements, the sample solutions were contained in a $1 \mathrm{~cm}$ quartz cell and their absorbance at the excitation wavelength was around 0.1. For FU measurements, the sample solutions were enclosed in a $0.4 \mathrm{~mm}$ rotating cell and their absorbance at 400 $\mathrm{nm}$ was about 0.1 . For TA, the solutions were in a $1 \mathrm{~mm}$ thick quartz cell, had an absorbance around 0.5 at $400 \mathrm{~nm}$ and were continuously stirred by $\mathrm{N}_{2}$ bubbling. For the other experiments, aerated solutions were used. No significant sample degradation was observed after the measurements. 


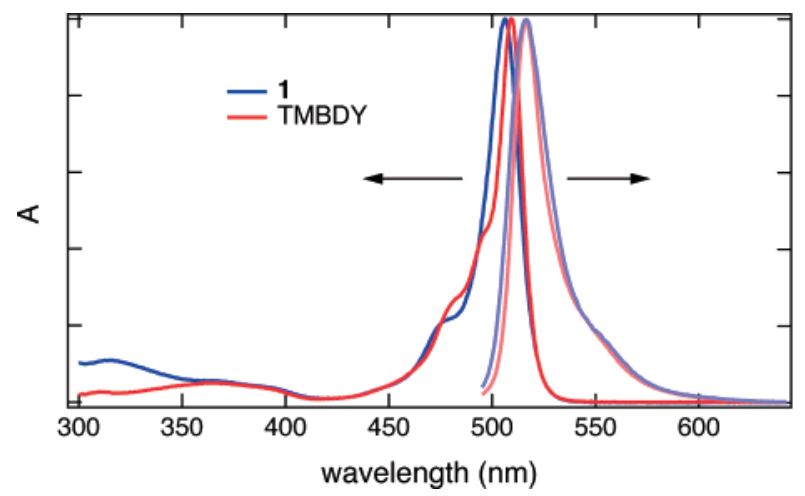

Figure 2. Absorption and fluorescence spectra of $\mathbf{1}$ and TMBDY in toluene (excitation at $480 \mathrm{~nm}$ ).

TABLE 1: Photophysical Properties of the DABS and of TMBDY in Toluene

\begin{tabular}{lcccccc}
\hline molecule & $\begin{array}{c}\lambda_{\mathrm{a}} \\
(\mathrm{nm})^{a}\end{array}$ & $\begin{array}{c}\lambda_{\mathrm{f}} \\
(\mathrm{nm})^{a}\end{array}$ & $\begin{array}{c}E\left(\mathrm{~S}_{1}\right) \\
(\mathrm{eV})^{b}\end{array}$ & $\Phi_{\mathrm{f}}{ }^{c}$ & $\begin{array}{c}\tau_{\mathrm{f}} \\
(\mathrm{ns})^{d}\end{array}$ & $\begin{array}{c}k_{\mathrm{rad}} \\
\left(\mathrm{s}^{-1}\right)^{e}\end{array}$ \\
\hline $\mathbf{1}$ & 506 & 518 & 2.42 & 0.39 & 3.32 & $1.17 \times 10^{8}$ \\
$\mathbf{2}$ & 517 & 532 & 2.36 & 0.39 & 3.24 & $1.20 \times 10^{8}$ \\
$\mathbf{3}$ & 519 & 534 & 2.35 & 0.24 & 2.25 & $1.07 \times 10^{8}$ \\
$\mathbf{4}$ & 534 & 550 & 2.29 & 0.19 & 1.70 & $1.11 \times 10^{8}$ \\
TMBDY & 509 & 518 & 2.41 & 0.73 & 4.90 & $1.49 \times 10^{8}$
\end{tabular}

${ }^{a}$ Absorption and fluorescence maxima. ${ }^{b}$ Excited-state energy. ${ }^{c}$ Fluorescence quantum yield. ${ }^{d}$ Fluorescence lifetime. ${ }^{e}$ Radiative rate constant.

Quantum Chemistry Calculations. The ground-state gasphase geometries of $\mathbf{1 - 4}$ were fully optimized at the density functional level of theory (DFT) using the B3LYP functional ${ }^{51,52}$ and a [3s2p1d] basis set. ${ }^{53}$ The calculations were carried out using Turbomole version 5.8.0..$^{54,55}$

\section{Results}

Steady-State Measurements. The steady-state absorption and emission spectra of $\mathbf{1}$ and TMBDY in toluene are shown in Figure 2. The absorption spectra of both compounds are quite similar down to $350 \mathrm{~nm}$, except for a small difference in the location of the absorption maximum and in the relative intensity of the shoulders, which have been ascribed to a vibronic transition. ${ }^{45}$ The small band centered around $315 \mathrm{~nm}$, which is also present in the absorption spectra of the other DABS but which is absent with TMBDY, is most probably due to the dinitro-triptycene group. The fluorescence spectra of TMBDY and all DABS have a very similar shape. As indicated in Table 1 , both absorption and fluorescence spectra exhibit a red shift of about $500 \mathrm{~cm}^{-1}$ and $1000 \mathrm{~cm}^{-1}$ upon addition of one and two, respectively, $\mathrm{Br}$ atoms on the chromophoric part of the DABS. The similarity between the spectra of TMBDY and of the DABS indicates a weak perturbation of the BODIPY chromophore upon substitution at the 8-position. Table 1 shows that the fluorescence quantum yields, $\Phi_{\mathrm{f}}$, in toluene are substantially smaller for the DABS than for TMBDY. Moreover, addition of $\mathrm{Br}$ atoms also leads to a reduction of the fluorescence quantum yield.

A quantitative determination of $\Phi_{\mathrm{f}}$ in polar solvents was not performed. However, the fluorescence intensities of TMBDY and of $\mathbf{4}$ were found to be essentially the same in polar solvents, whereas those of the other DABS, especially $\mathbf{1}$, were strongly reduced.

Isotropic Time-Resolved Fluorescence Measurements. The fluorescence decay of TMBDY and of all DABS in toluene can

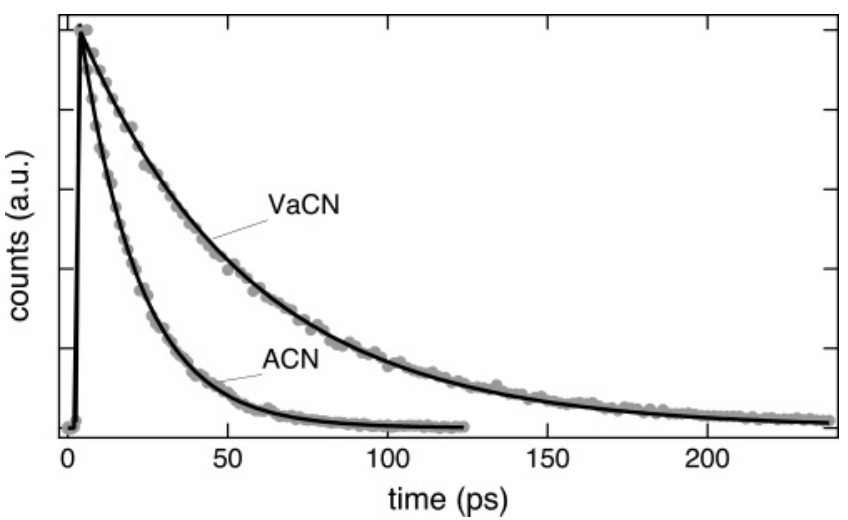

Figure 3. Time profiles of the fluorescence of $\mathbf{1}$ in $\mathrm{ACN}$ and $\mathrm{VaCN}$ measured at $525 \mathrm{~nm}$ after excitation at $400 \mathrm{~nm}$ and best singleexponential fits (solid lines).

TABLE 2: Fluorescence Lifetime, $\tau_{\mathrm{f}}$ (in ps), Measured with 1-3 in Various Polar Solvents and Time Constants of Charge Separation, $\tau_{\mathrm{CS}}($ in ps), Determined from Eq 2

\begin{tabular}{lcrrrr}
\hline solvent & $\tau_{\mathrm{f}}(\mathbf{1}) \approx \tau_{\mathrm{CS}}(\mathbf{1})$ & $\tau_{\mathrm{f}}(\mathbf{2})$ & $\tau_{\mathrm{CS}}(\mathbf{2})$ & $\tau_{\mathrm{f}}(\mathbf{3})$ & $\tau_{\mathrm{CS}}(\mathbf{3})$ \\
\hline $\mathrm{ACN}$ & 18 & 136 & 142 & 156 & 168 \\
$\mathrm{PrCN}$ & 27.5 & 387 & 439 & 467 & 590 \\
$\mathrm{BuCN}$ & 38 & & & & \\
$\mathrm{VaCN}$ & 51.5 & 1210 & 1930 & 1290 & 3023 \\
$\mathrm{DCM}$ & 14.5 & 298 & 328 & 465 & 586 \\
$\mathrm{CHCl}$ & 26 & 327 & 364 & & \\
$\mathrm{DCE}$ & 33 & $675^{a}$ & 853 & $990^{b}$ & 1770
\end{tabular}

${ }^{a}$ A 1.34 ns component with a relative amplitude of 0.3 is also present. ${ }^{b}$ A $1.76 \mathrm{~ns}$ component with a relative amplitude of 0.12 is also present.

be very well reproduced by an exponential function with a time constant, $\tau_{\mathrm{f}}$, of several nanoseconds (see Table 1 ). The radiative rate constants, $k_{\text {rad }}$, calculated from these lifetimes and the fluorescence quantum yields are essentially the same for all DABS. Consequently, the smaller quantum yields measured with $\mathbf{3}$ and $\mathbf{4}$ are due to an enhanced nonradiative decay channel, most probably intersystem crossing (isc). The difference between $\mathbf{2}$ and $\mathbf{3}$ is surprising, as both possess a single $\mathrm{Br}$ substituent. One possible reason is that the $\mathrm{Br}$ atom in $\mathbf{3}$ is rather close to a nitro group, which is known to favor isc. ${ }^{56-59}$ This proximity might increase spin-orbit coupling and thus result in a faster isc. This effect, together with the presence of a second $\mathrm{Br}$ atom, could also explain the shorter fluorescence lifetime measured with 4.

The smaller fluorescence quantum yield of the DABS relative to that of TMBDY is partially due to a somewhat smaller radiative rate constant. This could be explained by the weak perturbation of the BODIPY chromophore upon substitution at the 8-position, which is also responsible for the differences observed in the absorption and fluorescence spectra.

The fluorescence dynamics of both TMBDY and $\mathbf{4}$ were also measured in $\mathrm{ACN}, \mathrm{BuCN}$, and $\mathrm{CHCl}_{3}$ and were found to be essentially the same as in toluene, in agreement with the similar steady-state fluorescence intensity. The decay times found for TMBDY are in very good agreement with those reported in the literature. ${ }^{28}$

The fluorescence lifetimes of $\mathbf{1 - 3}$ in weakly to strongly polar solvents are listed in Table 2 . The time constants below $300 \mathrm{ps}$ have been determined from FU measurements, while the larger ones have been obtained from TCSPC experiments. Except for $\mathbf{2}$ and $\mathbf{3}$ in DCE, all decays could be very well reproduced by an exponential function as shown in Figure 3 for $\mathbf{1}$ in ACN 


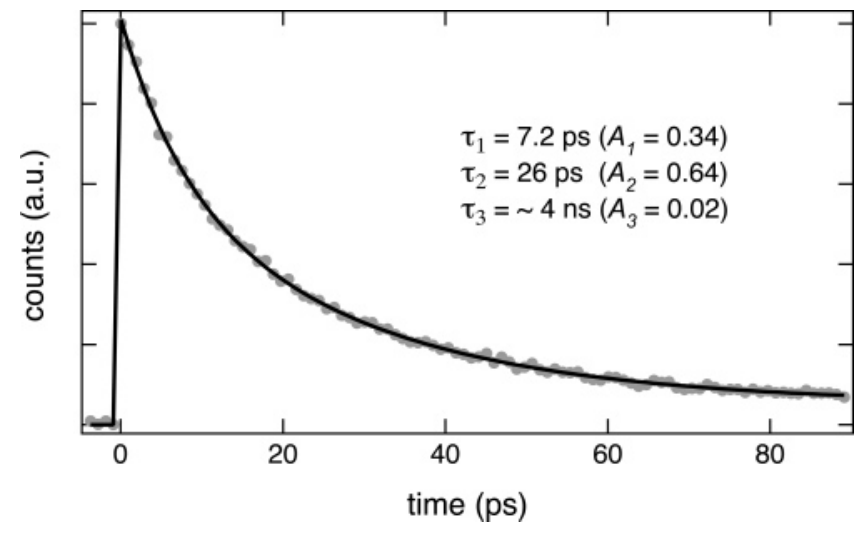

Figure 4. Time profile of the fluorescence of TMBDY in nitrobenzene measured at $540 \mathrm{~nm}$ after excitation at $400 \mathrm{~nm}$ and best triexponential fit.

and $\mathrm{VaCN}$. No significant wavelength dependence of the fluorescence dynamics that could be ascribed to vibrational cooling or solvent-induced Stokes shift was observed, at least between 515 and $550 \mathrm{~nm}$. Wavelengths out of this range were not investigated.

It appears from Table 2 that the fluorescence lifetimes are the shortest with $\mathbf{1}$, independent of the solvent. On the other hand, the lifetimes of $\mathbf{2}$ and $\mathbf{3}$ are approximately 10-20 times larger. Although the decay times of $\mathbf{2}$ and $\mathbf{3}$ are of similar order of magnitude, those of $\mathbf{3}$ are systematically larger.

In order to investigate whether the substantial reduction of fluorescence lifetime of $\mathbf{1 - 3}$ in polar solvents is compatible with a nonradiative deactivation due to intramolecular ET to the nitrobenzene moieties, the fluorescence dynamics of TMBDY was measured in pure nitrobenzene. Figure 4 shows the time profile of the fluorescence intensity of TMBDY at 540 $\mathrm{nm}$ in nitrobenzene upon excitation at $400 \mathrm{~nm}$. This decay is dominated by two components with 7.2 and 26 ps time constants and with relative amplitudes of 0.34 and 0.64 , respectively. A very weak nanosecond component $(\approx 4 \mathrm{~ns})$ that might be due to impurities was also found. These short decay times compare rather well with that found with $\mathbf{1}$ in $\mathrm{ACN}$, whose dielectric constant is similar to that of nitrobenzene.

The fluorescence lifetime of $\mathbf{4}$ was also measured in nitrobenzene. By contrast to TMBDY, 4 exhibits a single-exponential decay with a time constant of $1.6 \mathrm{~ns}$. This indicates rather clearly that both intra- and intermolecular charge separation (CS) between the dibromosubstituted BODIPY chromophore and nitrobenzene are not operative.

Transient Absorption Measurements. Figure 5A shows TA spectra recorded with $\mathbf{1}$ in $\mathrm{BuCN}$ at different time delays after excitation at $500 \mathrm{~nm}$. The spectra are dominated by a band with negative intensity centered at $507 \mathrm{~nm}$ at early times and shifting to about $502 \mathrm{~nm}$ at later times. This spectral feature is due to both stimulated emission, responsible for the red side of the band, and bleach of the ground-state absorption, accounting for the blue side of the band. The blue shift of the transient spectrum can be explained by a faster decay of the stimulated emission compared to the ground-state recovery (GSR). No transient band that could be ascribed to the radical cation of the BODIPY chromophore is visible in these spectra. The absorption spectrum of an electrochemically oxidized solution of a similar BODIPY chromophore with a trimethoxybenzene at the 8-position exhibits a relatively weak absorption band centered at $513 \mathrm{~nm}$ that was assigned to the radical cation of the BODIPY moiety. ${ }^{44}$ Therefore, the contribution of the BODIPY cation is most probably totally hidden by the bleach and the stimulated

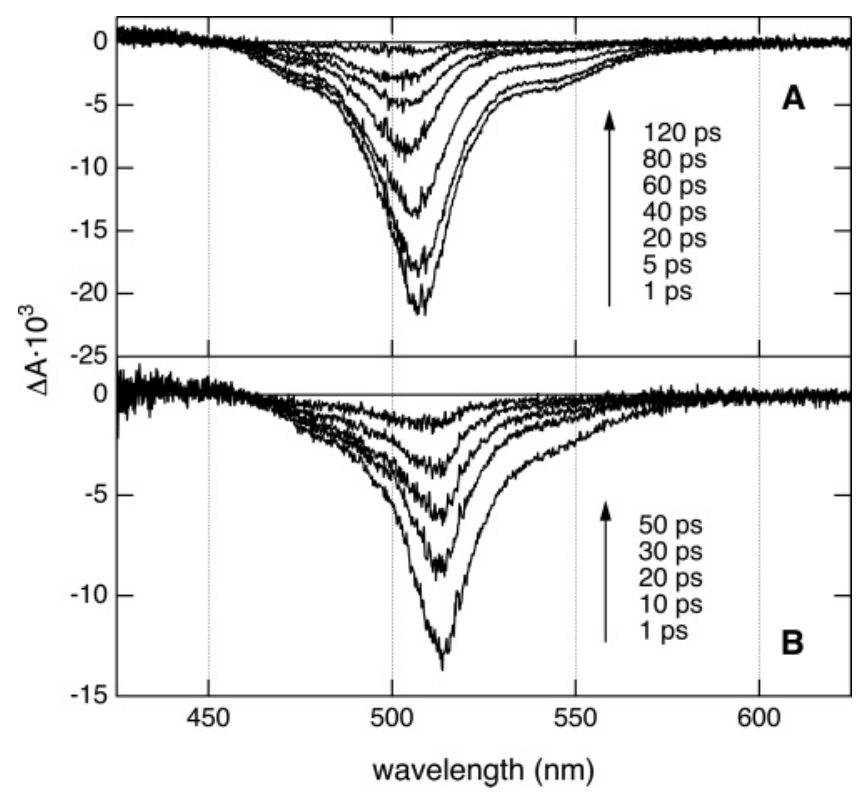

Figure 5. Transient absorption spectra measured with (A) 1 in $\mathrm{BuCN}$ and (B) TMBDY in nitrobenzene at different time delays after excitation at $500 \mathrm{~nm}$.

emission. The TA spectra also exhibit a weak and positive feature below $460 \mathrm{~nm}$ that corresponds to an absorption. The location of this band coincides rather well with that of the nitrobenzene radical anion reported in literature. ${ }^{60}$ The signal intensity at $530 \mathrm{~nm}$, which is dominated by stimulated emission, goes to zero with a time constant of about $35 \mathrm{ps,} \mathrm{in} \mathrm{very} \mathrm{good}$ agreement with the 38 ps value obtained by FU. The signal intensity at $500 \mathrm{~nm}$, due essentially to ground-state bleaching, evolves with a time constant of 52 ps. These two different time constants confirm the involvement of a third state, namely, a charge-separated state (CS state), additionally to the locally excited state (LE state) and to the ground state. In principle, the population of these three states should be governed by a kinetics for two consecutive first-order reactions, namely, CS from the LE to the CS state and charge recombination (CR) from the CS to the ground state. The well-known solutions of the corresponding differential equations are given in the Supporting Information. Although the number of recorded TA spectra was small, a global analysis was tentatively performed using these solutions as trial functions and the CS time constant obtained from the FU fluorescence as well as the steady-state absorption spectrum of $\mathbf{1}$ in BuCN as fixed parameters (see the Supporting Information for details). A CR time constant of 27 ps, e.g., considerably smaller than that for CS, was obtained from this analysis. Moreover, the resulting spectrum associated with the CS state consists in a small band with a maximum around $514 \mathrm{~nm}$ (see Figure S1, Supporting Information). However, this spectrum has to be considered with great caution first because the transient spectrum of the LE state is not known and thus that obtained from the global analysis cannot be confirmed and second because the number transient spectra is not sufficient to really resolve the departure from exponential kinetics that is expected for the population of the final state in a reaction with two consecutive first-order processes. On the other hand, the 27 ps CR time constant obtained from this analysis can be regarded with better confidence. Indeed, simulated GSR dynamics assuming a 38 ps CS time constant and a 30 ps CR time constant could be well reproduced using an exponential function with a 52 ps time constant, as found experimentally (Figure S2, Supporting Information). 


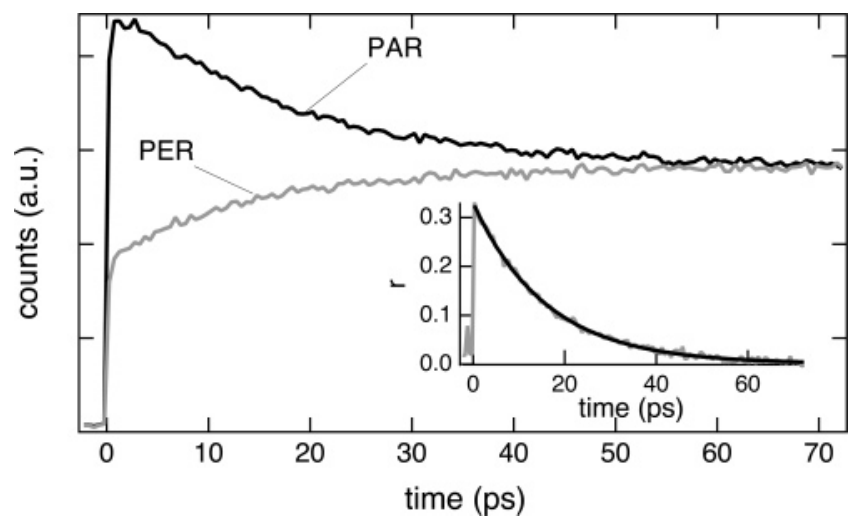

Figure 6. Time evolution of the polarized fluorescence measured with TMBDY in ACN at $525 \mathrm{~nm}$ after excitation at $400 \mathrm{~nm}$. The inset shows the decay of the polarization anisotropy.

TABLE 3: Fluorescence Anisotropy Decay Times, $\tau_{\mathrm{r}}$, of TMBDY in Solvents of Various Viscosities, $\eta$

\begin{tabular}{ccc}
\hline solvent & $\eta(\mathrm{cP})^{a}$ & $\tau_{\mathrm{r}}(\mathrm{ps})$ \\
\hline $\mathrm{ACN}$ & 0.35 & 16.0 \\
PrCN & 0.41 & 19.3 \\
$\mathrm{DCM}$ & 0.43 & 19.8 \\
$\mathrm{THF}$ & 0.55 & 21.8 \\
$\mathrm{CHCl}$ & 0.56 & 28.3 \\
$\mathrm{BuCN}$ & 0.58 & 25.8 \\
TOL & 0.59 & 20.7 \\
VaCN & 0.74 & 30.9 \\
DCE & 0.84 & 35.7 \\
BzCN & 1.30 & 48.5 \\
$a$ From ref 61. & &
\end{tabular}

Thus in BuCN, both CS and CR in $\mathbf{1}$ take place on a similar time scale. Because of this, the population of the CS state remains always relatively small and is therefore difficult to detect, especially if its absorption spectrum overlaps with those of other populations.

The TA spectra in ACN are essentially the same as in BuCN. However, the GSR time constant is shorter and amounts to 25 ps. With the use of the CS time constant of 18 ps obtained from the FU measurements, such a 25 ps GSR time can be reproduced by assuming a CR time constant of about 17 ps.

Figure 5B shows that the TA spectra recorded with TMBDY in pure nitrobenzene are very similar to those obtained with $\mathbf{1}$ in both BuCN and ACN. The small TA band around $450 \mathrm{~nm}$ that can be ascribed to nitrobenzene anion is also visible. However, its intensity is too small to extract any kinetic information. The bleach recovery at $500 \mathrm{~nm}$ and the decay of the stimulated emission at $530 \mathrm{~nm}$ can be reasonably well reproduced by exponential functions with time constants of 30 and $15 \mathrm{ps}$, respectively. The latter value is in good agreement with the average lifetime of 19 ps obtained by FU. From these values, the CR time constant can be estimated to be of the order of 20-25 ps. In this case again, CS and CR occur on a similar time scale.

TA measurements were also performed with $\mathbf{2}$ and $\mathbf{3}$ in ACN. In both cases, the spectra are dominated by a band with negative intensity centered at 515 and $520 \mathrm{~nm}$, respectively, and due to both stimulated emission and ground-state absorption bleach as well. However, contrary to $\mathbf{1}$ and TMBDY, the position of this band does almost not shift with time. Moreover, the kinetics at both sides of the band are the same within the experimental error and agree with those measured by FU (see Table 2). This indicates that $\mathrm{CR}$ in $\mathbf{2}$ and $\mathbf{3}$ in $\mathrm{ACN}$ should be at least 4-5 times faster than CS, which occurs with a time constant of about

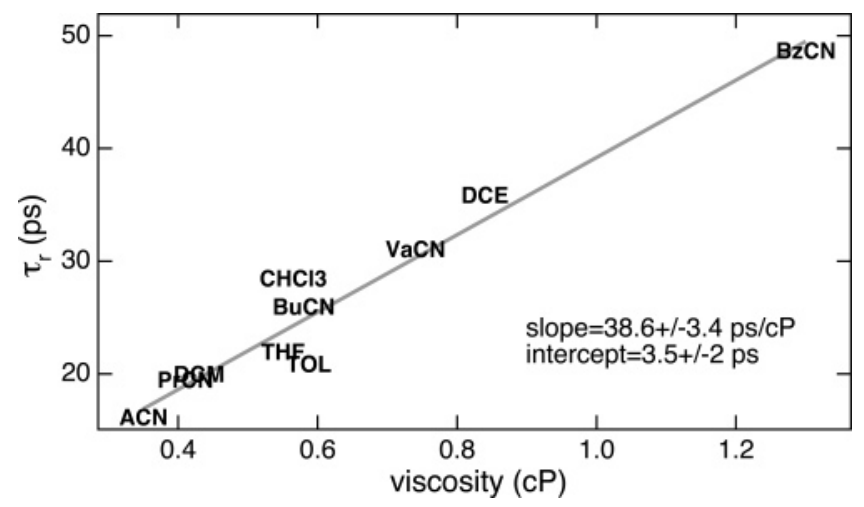

Figure 7. Viscosity dependence of the fluorescence anisotropy decay time of TMBDY and best linear fit (solid gray line).

140 ps. Consequently, the time scale of CR in these two DABS do not differ very much from that measured with $\mathbf{1}$.

Anisotropic Time-Resolved Fluorescence Measurements. As stated above, the relative orientation of the BODIPY chromophore and the nitrobenzene moieties in the DABS can vary upon rotation about the single bond in the 8-position. In order to determine the time scale associated with this motion, the reorientational dynamics of TMBDY has been determined by measuring the decay of its fluorescence polarization anisotropy in various solvents. Figure 6 shows the time evolution of the polarized fluorescence of TMBDY in ACN measured by FU. The inset shows that the anisotropy, $r$, decays exponentially from an initial value of 0.33 to 0 with a time constant, $\tau_{\mathrm{r}}$, of 16 ps. This time constant corresponds to the reorientation of TMBDY by rotational diffusion. Similar measurements have been performed in other solvents of various viscosities, and the resulting anisotropy decay times are listed in Table 3. Figure 7 indicates that $\tau_{\mathrm{r}}$ increases linearly with viscosity, in agreement with the following modified Stokes-Einstein-Debye equation: ${ }^{62,63}$

$$
\tau_{\mathrm{r}}=\tau_{\mathrm{r} 0}+\frac{f C V \eta}{k_{\mathrm{B}} T}
$$

where $\tau_{\mathrm{r} 0}$ is the zero-viscosity intercept, $k_{\mathrm{B}}$ is the Boltzmann constant, $T$ is the temperature, and $f$ is a shape factor that depends on the axis ratio of the ellipsoid. For TMBDY, which can be considered as a prolate ellipsoid with an axis ratio of 1.5 and with the transition dipole moment oriented along the long axis, $f$ can be estimated to be around 1.2. The volume of the rotating body, $V$, calculated using the van der Waals increments method, ${ }^{64,65}$ is equal to $213 \AA^{3}$. Finally, the boundary condition parameter, $C$, amounts to unity for stick hydrodynamics, i.e., when molecules of the first solvent shell follow the reorientational motion of the solute. This condition is often found with polar molecules in polar solvents. ${ }^{66-68}$ For slip hydrodynamics, the value of $C$ depends on the axis ratio of the rotating ellipsoid and should amount to 0.11 for a molecule like TMBDY. ${ }^{69}$ The slope obtained from a linear fit to the data shown in Figure 7 is $38.6 \pm 3.4 \mathrm{ps} / \mathrm{cP}$, while the intercept is $3.5 \pm 2$ ps. The physical meaning of this intercept has been discussed in detail in ref 70. The $C$ value obtained from the slope and using the $f$ and $V$ calculated above amounts to 0.64 . This indicates solute-solvent interactions of moderate strength, in reasonable agreement with the relatively small permanent electric dipole moment of $3.2 \mathrm{D}$ in both $\mathrm{S}_{0}$ and $\mathrm{S}_{1}$ states. ${ }^{71}$

The decay of the anisotropy has also been measured with $\mathbf{1}$ in toluene, and the corresponding time constant amounts to 73 
ps. This is larger by a factor 3.4 compared to that of TMBDY, whereas the volume of $\mathbf{1}$ is only larger by a factor 2.3. This apparent discrepancy is probably due to differences in shape factor and boundary condition. Measurements were not performed in other solvents because the fluorescence lifetime of $\mathbf{1}$ is smaller that its anisotropy decay time. Finally, the anisotropy decay time of 84 ps measured with 4 in toluene agrees well with a volume increase by a factor of about 1.1 by going from 1 to 4 .

\section{Discussion}

The strong reduction of the fluorescence lifetime of $\mathbf{1}-\mathbf{3}$ by going from toluene to more polar solvents can be rather unambiguously ascribed to the occurrence of an intramolecular CS between the BODIPY chromophore and the nitrobenzene moieties of the DABS. Although the CS state could not be clearly seen in the TA spectra, apart from the small $450 \mathrm{~nm}$ band most probably due to the nitrobenzene anion, the ultrashort time scale of the process measured with $\mathbf{1}$ and its occurrence in polar solvents only point toward ET. Similarly fast CS has been reported for a BODIPY chromophore directly linked to a trimethoxybenzene. ${ }^{44}$ The occurrence of CS is further supported by energetic considerations. Indeed, the driving force for CS, $\Delta G_{\mathrm{CS}}$, between TMBDY in the first singlet excited state and nitrobenzene, calculated as $\Delta G_{\mathrm{CS}}=-E\left(\mathrm{~S}_{1}\right)+e\left[E_{\mathrm{ox}}(\mathrm{D})-E_{\mathrm{red}^{-}}\right.$ (A)], amounts to $-0.15 \mathrm{eV}$, using $E_{\mathrm{OX}}(\mathrm{D})=1.11 \mathrm{~V}$ versus $\mathrm{SCE}$ for TMBDY, ${ }^{44} E_{\text {red }}(\mathrm{A})=-1.15 \mathrm{~V}$ versus SCE for nitrobenzene, ${ }^{72}$ and with the energy of the $S_{1}$ state, $E\left(S_{1}\right)$, determined from the absorption and fluorescence spectra (see Table 1). The Coulombic term, $C$, has been omitted in the calculation of $\Delta G_{\mathrm{CS}}$ because its magnitude in polar solvents is predicted to be negligibly small, $|C|<0.05 \mathrm{eV}$. This relatively weak driving force in polar solvents could explain why CS is no longer operative in toluene. The CS time constant in the DABS in a given polar solvent, $\mathrm{P}$, can be estimated by comparing the fluorescence lifetimes measured in this solvent and in toluene:

$$
\tau_{\mathrm{CS}}{ }^{-1}(\mathrm{P}) \approx \tau_{\mathrm{f}}^{-1}(\mathrm{P})-\tau_{\mathrm{f}}^{-1}(\mathrm{TOL})
$$

The use of this equation is reasonable as the fluorescence lifetime of $\mathbf{1}$ in toluene is rather close to that of TMBDY. The $\tau_{\mathrm{CS}}$ values calculated with eq 2 are listed in Table 2 . As the fluorescence lifetime of $\mathbf{1}$ in polar solvents is much smaller than that in toluene, $\tau_{\mathrm{CS}}(\mathbf{1}) \approx \tau_{\mathrm{f}}(\mathbf{1})$.

Addition of a $\mathrm{Br}$ atom on the BODIPY chromophore lowers the energy of the $S_{1}$ state as shown in Table 1 and can additionally be expected to raise its oxidation potential. Both these effects reduce the driving force for CS. This explains why the excited-state decay of $\mathbf{1}$ in polar solvents is always faster than those of $\mathbf{2}$ and $\mathbf{3}$ and why CS is not operative with $\mathbf{4}$. It also agrees with the measurements performed in pure nitrobenzene.

The most striking feature in the above results is most certainly the solvent dependence of the fluorescence decay time of $\mathbf{1 - 3}$. As shown in Table 2, there is no apparent correlation between $\tau_{\mathrm{f}}$ or $\tau_{\mathrm{CS}}$ and the solvent polarity. Indeed, $\tau_{\mathrm{CS}}(\mathbf{1})$ is the shortest in ACN and DCM, which have very different dielectric constants $\left(\epsilon_{\mathrm{s}}(\mathrm{ACN})=37.5\right.$ and $\left.\epsilon_{\mathrm{s}}(\mathrm{DCM})=8.9\right) .{ }^{61} \mathrm{It}$ is also interesting to note that the $\tau_{\mathrm{CS}}$ values with $\mathbf{2}$ and $\mathbf{3}$ correlate quite well with those obtained with $\mathbf{1}$, showing that the CS process in all three DABS is governed by a similar solvent dependence.

Figure 8 shows that $\tau_{\mathrm{CS}}(\mathbf{1})$ correlates with the fluorescence anisotropy decay time of TMBDY, $\tau_{\mathrm{r}}$. The solvents can be separated into two groups, namely, the nitriles and the chlorinated solvents with THF, in both of which $\tau_{\mathrm{CS}}(\mathbf{1})$ varies linearly

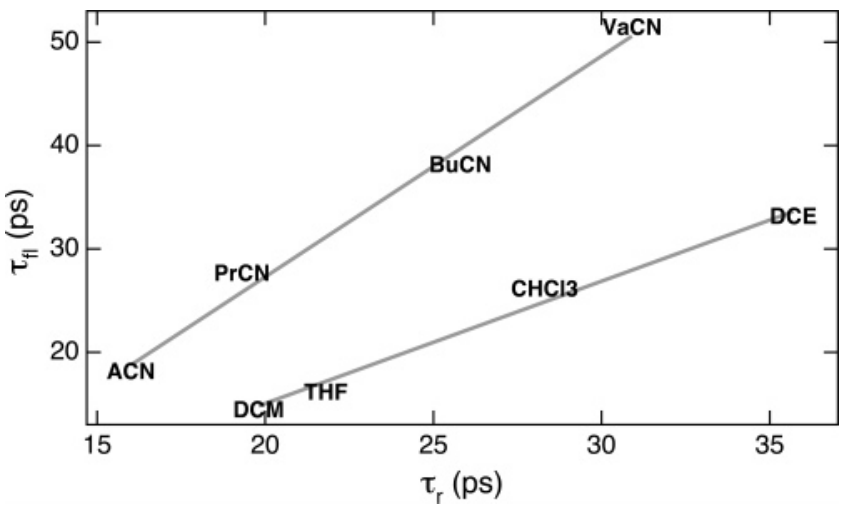

Figure 8. Correlation between the CS time constant of $\mathbf{1}, \tau_{\mathrm{CS}}$, and the fluorescence anisotropy decay time of TMBDY, $\tau_{\mathrm{r}}$, and best linear fits (solid gray lines).

with $\tau_{\mathrm{r}}$. The slope with the nitriles is around 2, whereas that with the other group of solvents amounts to 1.2. A similar but more scattered correlation can also be found between $\tau_{\mathrm{CS}}$ and solvent viscosity. The dynamics of solvation, especially that occurring through diffusive motion, is also related to viscosity. Given that for the present systems the stabilization of the CS state by solvation is crucial, a correlation between $\tau_{\mathrm{CS}}$ and the solvation time as predicted by theory, ${ }^{73}$ and as recently reported for intramolecular charge-transfer processes ${ }^{74}$ would not be surprising. A plot of $\tau_{\mathrm{CS}}$ as a function of the diffusive solvation times reported for the nitriles ${ }^{75,76}$ shows a reasonable correlation. However such a correlation is totally absent with the chlorinated solvents. ${ }^{75,77}$ An even more scattered plot is obtained when using the average solvation times, i.e., when taking the inertial solvent response, which is not related to viscosity, into account.

The optimized ground-state geometry of $\mathbf{1 - 3}$ in the gas phase indicates that the BODIPY chromophore is essentially orthogonal to the phenyl group in the 8-position. Indeed, the dihedral angle, $\theta_{\mathrm{d}}$, between these two moieties (defined by the bonds marked with a star in Figure 1) amounts to $91^{\circ}$ in $\mathbf{1}$ and $\mathbf{2}$ and to $89^{\circ}$ in $\mathbf{3}$. Figure 9 shows the frontier molecular orbitals of $\mathbf{1}$. For $\mathbf{2}$ and $\mathbf{3}$, these orbitals are qualitatively the same. The $\mathrm{HOMO}$ and LUMO+2 of $\mathbf{1}$ are essentially identical to the HOMO and LUMO of TMBDY. On the other hand, the LUMO and $\mathrm{LUMO}+1$ of $\mathbf{1}$ are almost exclusively centered on the nitrobenzene groups, with the LUMO on that in anti position relative to the BODIPY chromophore and the LUMO+1 on that in syn position. The strong similarity of the absorption and emission spectra of $\mathbf{1}$ and TMBDY suggests that excitation is localized on the BODIPY moiety. The $\mathrm{S}_{1}$ state of TMBDY has been shown to be well described as a HOMO-LUMO oneelectron transition. ${ }^{78}$ By analogy, the LE state of $\mathbf{1}$ can be reasonably assumed to be essentially populated through a oneelectron HOMO-LUMO+2 transition. The fluorescence quenching should thus be associated with a charge redistribution from the BODIPY-centered LUMO+2 to the nitrobenzene-centered $\mathrm{LUMO}$ and $\mathrm{LUMO}+1$.

The geometry of these molecules suggests that both throughbond and through-space CS could be operative. The throughbond pathway requires the coupling of the LUMO+2 and the lower LUMOs via empty orbitals of the bridge, namely, the phenyl ring directly linked to the BODIPY moiety. However, because of the orthogonality of these two units, this superexchange interaction should be quite small, and consequently the resulting electronic coupling constant, $V_{\mathrm{DA}}$, should not favor ultrafast through-bond CS. However, a variation of the dihedral angle, $\theta_{\mathrm{d}}$, upon torsion of the single bond in the 8-position could substantially improve this electronic coupling. 

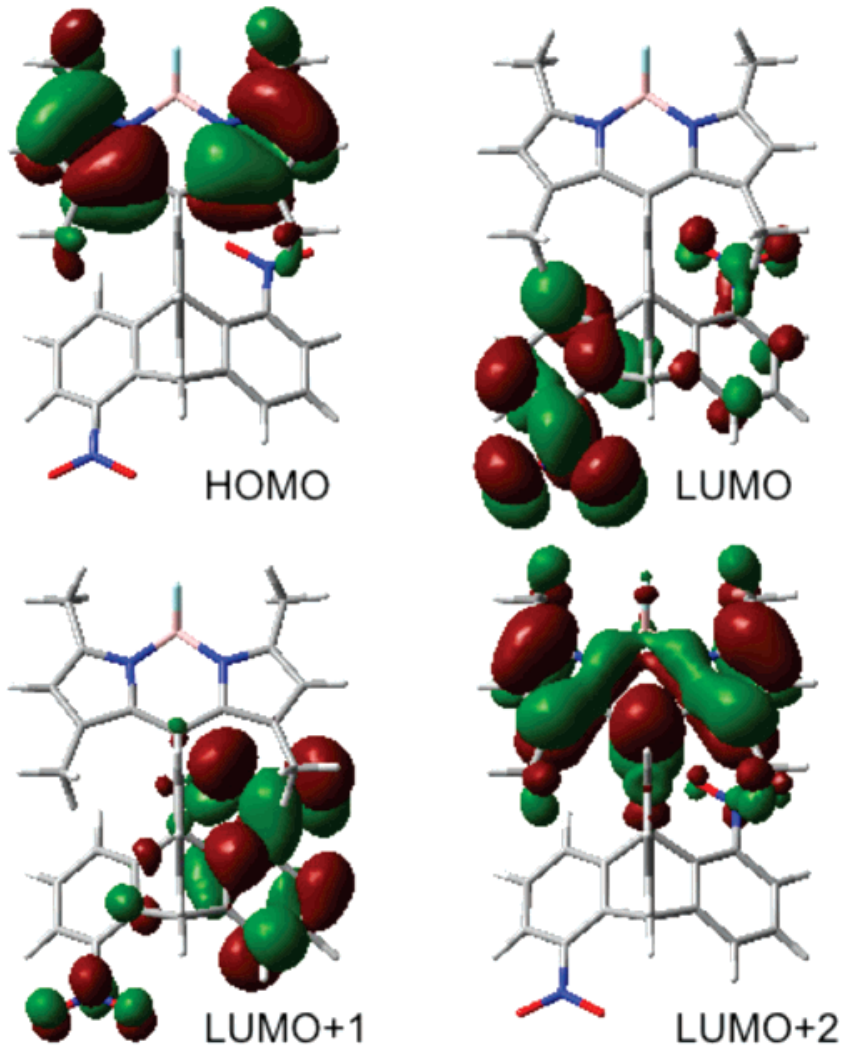

Figure 9. Frontier molecular orbitals of $\mathbf{1}$ computed at the B3LYP/ [3s 2 p1d] level of theory.

Through-space CS might occur from the LUMO+2 to the LUMO +1 localized on the closest nitrobenzene group. In the optimized geometry of $\mathbf{1}$, the distance between the nitrogen atom and the closest aromatic carbon atom of the BODIPY moiety, $d_{\mathrm{N}-\mathrm{C}}$ (see Figure 1), amounts to $4.1 \AA$. In this case again, torsional motion about the single bond in the 8-position leads to a reduction of this distance and thus to a larger electronic coupling for through-space transfer. With $\theta_{\mathrm{d}}=105^{\circ}$, the $d_{\mathrm{N}-\mathrm{C}}$ value is reduced to $3.5 \AA$. Consequently, torsional motion favors CS independently of the pathway.

The gas-phase energy of $\mathbf{1 - 3}$ has been calculated as a function of $\theta_{\mathrm{d}}$, the other coordinates being each time reoptimized. The energy required to twist 1 from $\theta_{\mathrm{d}}=91^{\circ}$ to $\theta_{\mathrm{d}}=$ $105^{\circ}$ amounts to $0.7 \mathrm{kcal} \cdot \mathrm{mol}^{-1}$ only. For comparison, the activation energy associated to a diffusional process like translation or rotation is typically of the order of $2.5 \mathrm{kcal} \cdot \mathrm{mol}^{-1}$. Therefore, the time scale of this quasi-free torsional motion in solution should be of the same order of magnitude as that of the reorientational motion of the BODIPY chromophore. This can thus explain the observed correlation between the CS time constant and the reorientational time of TMBDY.

As a consequence, the reaction coordinate for CS should be dominated by the dihedral angle, $\theta_{\mathrm{d}}$. The charge-transfer dynamics should thus be limited by torsional motion about the single bond in the 8-position. Mathematically, one could express the CS rate constant in an Arrhenius-type form, with the preexponential factor inversely proportional to the time constant associated with the torsional motion. This time constant should set the limit of the fastest possible CS, when the process is barrierless, as might be the case with $\mathbf{1}$. This is somewhat similar to the case of solvent-controlled adiabatic ET, where the rate constant is proportional to the inverse solvent relaxation time. ${ }^{73,79}$ The slower fluorescence quenching measured with both $\mathbf{2}$ and $\mathbf{3}$ can be due to several additive factors: (1) a slower torsional motion due to the larger hydrodynamic volume of the bromo-substituted BODIPY chromophore, (2) a slightly higher energy required to vary $\theta_{\mathrm{d}}$ because of the steric hindrance introduced by the bromine atoms, and (3) a smaller driving force for $\mathrm{CS}$, the latter factor being most certainly dominant. A smaller driving force implies a larger activation energy than for $\mathbf{1}$ where CS is probably barrierless and thus a slower CS. However, as the preexponential factor depends on friction, the solvent dependence observed with $\mathbf{1}$ is also present with $\mathbf{2}$ and $\mathbf{3}$.

We have no explanation for the presence of the two distinct linear dependencies of $\tau_{\mathrm{CS}}$ on $\tau_{\mathrm{r}}$ shown in Figure 8. A quite acceptable single linear correlation is obtained in a plot of $\tau_{\mathrm{CS}}$ versus $\tau_{\mathrm{r}} / \rho$, where $\rho$ is the density of the solvent. There is however no evident reason for such a correlation, which might be purely fortuitous. However, it should be noted that friction is certainly not the only effect of solvent on the CS dynamics in these DABS. As stated above, some solvation energy is required to make $\mathrm{CS}$ exergonic. As a consequence, $\mathrm{CS}$ in a low viscosity but apolar solvent is very slow or even nonoperative. The reorganization energy of the solvents used here can also be expected to differ substantially. All these various contributions might be at the origin of the two separate lines shown in Figure 8.

The two isomers $\mathbf{2}$ and $\mathbf{3}$ have not only different fluorescence lifetimes in nonpolar solvents (Table 1), but also different CS time constants in polar solvents (Table 2). The latter difference might be due to steric effects in $\mathbf{3}$ where the bromine atom is close to a nitro group. Indeed, the $d_{\mathrm{N}-\mathrm{C}}$ distance amounts to 4.05 and $4.23 \AA$ in the optimized equilibrium geometries of $\mathbf{2}$ and 3, respectively. Moreover, the energy required to increase the dihedral angle $\theta_{\mathrm{d}}$ up to $105^{\circ}$ is larger for $\mathbf{3}$ than for $\mathbf{2}$ by about $50 \%$. Both the larger $d_{\mathrm{N}-\mathrm{C}}$ value and the reduced ability for torsion should lead to a smaller electronic coupling for both through-bond and through-space CS.

Although it has only been measured in two solvents, namely, $\mathrm{ACN}$ and $\mathrm{BuCN}$, the $\mathrm{CR}$ dynamics in $\mathbf{1}$ occurs on the same time scale as CS and thus exhibits a similar solvent dependence. Such a dependence is expected as the electronic coupling for CR depends on the overlap of the HOMO and LUMO, which, as shown in Figure 8, are localized on the donor and acceptor parts of the DABS, respectively. Moreover, the driving force for $\mathrm{CR}$ can be expected to be larger in the less polar BuCN and, as $\mathrm{CR}$ takes place in the inverted regime, slower $\mathrm{CR}$ is predicted in this solvent. The driving force for $\mathrm{CR}$, calculated as $\Delta G_{\mathrm{CR}}=e\left[E_{\mathrm{red}}(\mathrm{A})-E_{\mathrm{ox}}(\mathrm{D})\right]$, amounts to about $-2.25 \mathrm{eV}$ in ACN. Time constants of the order of $10 \mathrm{ps}$ have been reported for the CR of geminate ion pairs with a similar driving force. ${ }^{80}$ The time scale of CR in $\mathbf{2}$ and $\mathbf{3}$ seems not to differ very much from that in $\mathbf{1}$. This could be interpreted as an evidence that $\Delta G_{\mathrm{CR}}$ is about the same for $\mathbf{1 - 3}$ and thus that the differences in driving force for $\mathrm{CS}$ upon addition of $\mathrm{Br}$ atoms are mostly due to differences in the excited-state energy.

\section{Concluding Remarks}

The DABS investigated here have revealed a very unusual solvent dependence of intramolecular CS dynamics. By considering their specific architecture, it appears that this dependence is most probably a manifestation of the interplay between the reorientation of the reactive parts and CS itself. When the driving force for CS is sufficiently large, as in the case of $\mathbf{1}$ in polar solvents, the process is limited by this reorientational motion. This has direct implication for bimolecular ET processes, where the relative orientation of the reaction partners has always been neglected. 
In previous reports, the BODIPY chromophores have been used as electron acceptors. The present investigation shows that these compounds can also be used very efficiently as electron donors. Moreover, according to their $S_{1}$ energy and redox potentials, photoinduced CS between two BODIPY dyes should also be operative. These dyes may thus prove to be valuable candidates for CS in multichromophoric assemblies.

Acknowledgment. The authors thank Professor A. Gossauer (Chemistry Department of the University of Fribourg) for samples of TMBDY and compounds 1-4 and Professor Laura Gagliardi (Department of Physical Chemistry of the University of Geneva) for her helpful advice concerning quantum chemistry calculations. This work was supported by the Fonds National Suisse de la Recherche Scientifique through Project No. 200020107466.

Supporting Information Available: Details on the global analysis of the TA spectra of $\mathbf{1}$ in BuCN and simulation of the ground-state recovery dynamics, including figures of the coefficients and the time evolution of various populations. This material is available free of charge via the Internet at http:// pubs.acs.org.

\section{References and Notes}

(1) Miller, J. R.; Calcaterra, L. T.; Closs, G. L. J. Am. Chem. Soc. 1984, 106, 3047.

(2) Closs, G. L.; Calcaterra, L. T.; Green, N. J.; Penfield, K. W.; Miller, J. R. J. Phys. Chem. 1986, 90, 3674.

(3) Oevering, H.; Paddon-Row, M. N.; Heppener, M.; Oliver, A. M.; Cotsaris, E.; Verhoeven, J. W.; Hush, N. S. J. Am. Chem. Soc. 1987, 109, 3258.

(4) Krongauz, V. V. J. Phys. Chem. 1992, 96, 2609.

(5) Jacques, P.; Allonas, X. Chem. Phys. Lett. 1995, 233, 533.

(6) Murata, S.; Tachiya, M. J. Phys. Chem. 1996, 100, 4064.

(7) Zhou, J.; Findley, B. R.; Braun, C. L.; Sutin, N. J. Chem. Phys. 2001, 114, 10448

(8) Marcus, R. A.; Sutin, N. Biochim. Biophys. Acta 1985, 811, 265.

(9) Burshtein, A. I. Adv. Chem. Phys. 2000, 114, 419.

(10) Wasielewski, M. R.; Niemczyk, M. P. J. Am. Chem. Soc. 1984, 106, 5043.

(11) Sakata, Y.; Tsue, H.; Goto, Y.; Misumi, S.; Asahi, T.; Nishikawa, S.; Okada, T.; Mataga, N. Chem. Lett. 1991, 1307.

(12) Maiya, B. G.; Doraiswamy, S.; Periasamy, N.; Venkataraman, B.; Krishnan, V. J. Photochem. Photobiol., A 1994, 81, 139.

(13) Sakata, Y.; Tsue, H.; O'Neil, M. P.; Wiederrecht, G. P.; Wasielewski, M. R. J. Am. Chem. Soc. 1994, 116, 6904.

(14) Yanagida, M.; Kanai, T.; Zhang, X.-Q.; Kondo, T.; Uosaki, K. Bull. Chem. Soc. Jpn. 1998, 71, 2555.

(15) Osuka, A.; Shin, J.-Y.; Yoneshima, R.; Shiratori, H.; Ohno, T.; Nozaki, K.; Nishimura, Y.; Yamazaki, I.; Taniguchi, S.; Shimizu, T.; Okada, T. J. Porphyrins Phthalocyanines 1999, 3, 729.

(16) Thomas, K. G.; Biju, V.; Guldi, D. M.; Kamat, P. V.; George, M. V. J. Phys. Chem. A 1999, 103, 10755.

(17) Armaroli, N.; Marconi, G.; Echegoyen, L.; Bourgeois, J.-P.; Diederich, F. Chem. Eur. J. 2000, 6, 1629.

(18) Guldi, D. M.; Luo, C.; Prato, M.; Troisi, A.; Zerbetto, F.; Scheloske, M.; Dietel, E.; Bauer, W.; Hirsch, A. J. Am. Chem. Soc. 2001, 123, 9166.

(19) Kreher, D.; Cariou, M.; Liu, S.-G.; Levillain, E.; Veciana, J.; Rovira, C.; Gorgues, A.; Hudhomme, P. J. Mater. Chem. 2002, 12, 2137.

(20) Van Hal, P. A.; Beckers, E. H. A.; Meskers, S. C. J.; Janssen, R. A. J.; Jousselme, B.; Blanchard, P.; Roncali, J. Chem. Eur. J. 2002, 8, 5415.

(21) Guldi, D. M.; Hirsch, A.; Scheloske, M.; Dietel, E.; Troisi, A.; Zerbetto, F.; Prato, M. Chem. Eur. J. 2003, 9, 4968.

(22) Beckers, E. H. A.; Meskers, S. C. J.; Schenning, A. P. H. J.; Chen, Z.; Wuerthner, F.; Marsal, P.; Beljonne, D.; Cornil, J.; Janssen, R. A. J. J. Am. Chem. Soc. 2006, 128, 649.

(23) Vauthey, E.; Phillips, D. Chem. Phys. 1990, 147, 421.

(24) Allonas, X.; Jacques, P. Chem. Phys. 1997, 215, 371.

(25) Castner, E. W., Jr.; Kennedy, D.; Cave, R. J. J. Phys. Chem. A 2000, 104, 2869.

(26) Morandeira, A.; Fürstenberg, A.; Gumy, J.-C.; Vauthey, E. J. Phys. Chem. A 2003, 107, 5375.

(27) Vauthey, E. J. Photochem. Photobiol., A 2006, 179, 1.
(28) Karolin, J.; Johansson, L. B. A.; Strandberg, L.; Ny, T. J. Am. Chem. Soc. 1994, 116, 7801.

(29) Burghart, A.; Kim, H.; Welch, M. B.; Thoresen, L. H.; Reibenspies, J.; Burgess, K. J. Org. Chem. 1999, 64, 7813.

(30) He, H.; Nunnally, B. K.; Li, L.-C.; McGown, L. B. Anal. Chem 1998, 70,3413

(31) Li, L.; McGown, L. B. J. Chromatogr., A 1999, 841, 95.

(32) Coskun, A.; Baytekin, B. T.; Akkaya, E. U. Tetrahedron Lett. 2003, 44,5649 .

(33) Koutaka, H.; Kosuge, J.-i.; Fukasaku, N.; Hirano, T.; Kikuchi, K. Urano, Y.; Kojima, H.; Nagano, T. Chem. Pharm. Bull. 2004, 52, 700.

(34) Gabe, Y.; Urano, Y.; Kikuchi, K.; Kojima, H.; Nagano, T. J. Am. Chem. Soc. 2004, 126, 3357.

(35) Yogo, T.; Urano, Y.; Ishitsuka, Y.; Maniwa, F.; Nagano, T. J. Am. Chem. Soc. 2005, 127, 12162.

(36) Sauer, M.; Arden-Jacob, J.; Drexhage, K. H.; Gobel, F.; Lieberwirth, U.; Muhlegger, K.; Muller, R.; Wolfrum, J.; Zander, C. Bioimaging 1998, 6,14 .

(37) Li, F.; Yang, S. I.; Ciringh, Y.; Seth, J.; Martin, C. H., III; Singh, D. L.; Kim, D.; Birge, R. R.; Bocian, D. F.; Holten, D.; Lindsey, J. S. J. Am. Chem. Soc. 1998, 120, 10001.

(38) Wagner, R. W.; Lindsey, J. S. J. Am. Chem. Soc. 1994, 116, 9759

(39) Wagner, R. W.; Lindsey, J. S.; Seth, J.; Palaniappan, V.; Bocian,

D. F. J. Am. Chem. Soc. 1996, $118,3996$.

(40) Wan, C.-W.; Burghart, A.; Chen, J.; Bergstroem, F.; Johansson, L. B. A.; Wolford, M. F.; Kim, T. G.; Topp, M. R.; Hochstrasser, R. M.; Burgess, K. Chem. Eur. J. 2003, 9, 4430.

(41) Huang, K.-H.; Wu, J.-Y.; Wu, W.-S.; Chang, C.-C.; Lee, H. L.; Chang, T.-C. J. Lumin. 2004, 107, 213.

(42) Ziessel, R.; Goze, C.; Ulrich, G.; Cesario, M.; Retailleau, P.; Harriman, A.; Rostron, J. P. Chem. Eur. J. 2005, 11, 7366.

(43) Torimura, M.; Kurata, S.; Yamada, K.; Yokomaku, T.; Kamagata, Y.; Kanagawa, T.; Kurane, R. Anal. Sci. 2001, 17, 155.

(44) Hattori, S.; Ohkubo, K.; Urano, Y.; Sunahara, H.; Nagano, T.; Wada, Y.; Tkachenko, N. V.; Lemmetyinen, H.; Fukuzumi, S. J. Phys. Chem. B 2005, 109, 15368

(45) Qin, W.; Baruah, M.; Van der Auweraer, M.; De Schryver, F. C.; Boens, N. J. Phys. Chem. A 2005, 109, 7371.

(46) Wilcox, C. F., Jr. J. Chem. Phys. 1960, 33, 1874.

(47) Bersohn, R.; Even, U.; Jortner, J. J. Chem. Phys. 1983, 79, 2163

(48) Kundu, T.; Nath, D.; Samanta, A.; Chowdhury, M. Chem. Phys. Lett. 1987, 133, 507.

(49) Morandeira, A.; Engeli, L.; Vauthey, E. J. Phys. Chem. A 2002 $106,4833$.

(50) Armagero, W. L. F.; Chai, C. L. L. Purification of Laboratory Chemicals; Elsevier: Amsterdam, 2003.

(51) Lee, C.; Yang, W.; Parr, R. G. Phys. Rev. B 1988, 37, 785.

(52) Becke, A. D. Phys. Rev. A 1988, 38, 3098.

(53) Schäfer, A.; Horn, H.; Ahlrichs, R. J. Chem. Phys. 1992, 97, 2571.

(54) Ahlrichs, R.; Bär, M.; Häser, M. Chem. Phys. Lett. 1989, 162, 165.

(55) Häser, M.; Ahlrichs, R. J. Comput. Chem. 1989, 10, 104.

(56) Hurley, R.; Testa, A. C. J. Am. Chem. Soc. 1968, 90, 1949.

(57) Hahn, R. C.; Johnson, R. P. J. Am. Chem. Soc. 1977, 99, 1508.

(58) Keana, J. F. W.; Cai, S. X. J. Org. Chem. 1990, 55, 3640.

(59) Chibisov, A. K.; Görner, H. J. Phys. Chem. A 1997, 101, 4305 .

(60) Shida, T. Electronic Absorption Spectra of Radical Ions; Elsevier: Amsterdam, 1988; Vol. Physical Sciences Data 34.

(61) Riddick, J. A.; Bunger, W. B. Organic Solvents; J. Wiley: New York, 1970.

(62) Alms, G. R.; Bauer, D. R.; Brauman, J. I.; Pecora, R. J. Chem. Phys. 1973, 59, 5310 .

(63) Fleming, G. R. Chemical Applications of Ultrafast Spectroscopy; Oxford University Press: New York, 1986.

(64) Bondi, A. J. Phys. Chem. 1964, 68, 441.

(65) Edward, J. T. J. Chem. Educ. 1970, 4, 261.

(66) von Jena, A.; Lessing, H. E. Ber. Bunsen-Ges. Phys. Chem. 1979, 83,181

(67) Vauthey, E. Chem. Phys. Lett. 1993, 216, 530.

(68) Williams, A. M.; Jiang, Y.; Ben-Amotz, D. Chem. Phys. 1994, 180, 119.

(69) Hu, C. M.; Zwanzig, R. J. Chem. Phys. 1974, 60, 4354.

(70) Evans, G. T.; Kivelson, D. J. Chem. Phys. 1986, 84, 385.

(71) Bergstroem, F.; Mikhalyov, I.; Haeggloef, P.; Wortmann, R.; Ny, T.; Johansson, L. B. A. J. Am. Chem. Soc. 2002, 124, 196.

(72) Jensen, B. S.; Parker, V. D. J. Chem. Soc., Chem. Commun. 1974, 10,367 .

(73) Rips, I.; Jortner, J. J. Chem. Phys. 1987, 87, 2090.

(74) Galievsky, V. A.; Druzhinin, S.; Demeter, A.; Jiang, Y.-B.; Kovalenko, S. A.; Lustres, J. L. P.; Venugopal, K.; Ernsting, N. P.; Allonas, X.; Noltemeyer, M.; Machinek, R.; Zachariasse, K. A. ChemPhysChem 2005, 6, 2307. 
(75) Horng, M. L.; Gardecki, J. A.; Papazyan, A.; Maroncelli, M. J. Phys. Chem. 1995, 99, 17311.

(76) Gumy, J. C.; Nicolet, O.; Vauthey, E. J. Phys. Chem. A 1999, 103, 10737.

(77) Iwami, Y.; Yamamoto, M.; Nishi, N.; Kakiuchi, T. Buseki Kagaku 2005, 54,485 .
(78) Kee, H. L.; Kirmaier, C.; Thamyongkit, P.; Youngblood, W. J.; Calder, M. E.; Ramos, L.; Noll, B. C.; Bocian, D. F.; Scheidt, W. R.; Birge, R. R.; Lindsey, J. S.; Holten, D. J. Phys. Chem. B 2005, 109, 20433.

(79) Zusman, L. D. Chem. Phys. 1980, 49, 295.

(80) Vauthey, E. J. Phys. Chem. A 2001, 105, 340. 